\title{
Genome-wide heritability analysis of severe malaria resistance reveals evidence of polygenic inheritance
}

\author{
Delesa Damena and Emile R. Chimusa*
}

\author{
Division of Human Genetics, Department of Pathology, Institute of Infectious Disease and Molecular Medicine \\ University of Cape Town, Private Bag, Rondebosch, 7700 Cape Town, South Africa.
}

${ }^{*}$ To whom correspondence should be addressed at. Division of Human Genetics, Department of Pathology, Institute of Infectious Disease and Molecular Medicine, University of Cape Town, Private Bag, Rondebosch, 7700, Cape Town, South Africa. Tel: +27 21406 6425;Email: emile.chimusa@.uct.ac.za. http://web.cbio.uct.ac.za/ emile/index.html

\begin{abstract}
Background: Estimating single nucleotide polymorphism (SNP)-heritability $\left(h^{2} g\right)$ of severe malaria resistance and its distribution across the genome might shed new light in to the underlying biology. Method: We investigated $h^{2} g$ of severe malaria resistance from a genome-wide association study (GWAS) dataset (sample size $=11657$ ). We estimated the $h^{2} \mathrm{~g}$ and partitioned in to chromosomes, allele frequencies and annotations using the genetic relationship-matrix restricted maximum likelihood approach. We further examined non-cell type-specific and cell type-specific enrichments from GWAS-summary statistics. Results: The $h^{2} g$ of severe malaria resistance was estimated at $0.21\left(\mathrm{se}=0.05, P=2.7 \times 10^{-5}\right), 0.20$ $\left(\mathrm{se}=0.05, \mathrm{P}=7.5 \times 10^{-5}\right)$ and $0.17\left(\mathrm{se}=0.05, \mathrm{P}=7.2 \times 10^{-4}\right)$ in Gambian, Kenyan and Malawi populations, respectively. A comparable range of $h^{2} g\left[0.21\left(\mathrm{se}=0.02, P<1 \times 10^{-5}\right)\right]$ was estimated from GWAS-summary statistics meta-analysed across the three populations. Partitioning analysis from raw genotype data showed significant enrichment of $h^{2} g$ in genic SNPs while summary statistics analysis suggests evidences of enrichment in multiple categories. Supporting the polygenic inheritance, the $h^{2} g$ of severe malaria resistance is distributed across the chromosomes and allelic frequency spectrum. However, the $h^{2} g$ is disproportionately concentrated on three chromosomes (chr 5, 11 and 20), suggesting cost-effectiveness of targeting these chromosomes in future malaria genomic sequencing studies. Conclusion: We report for the first time that the heritability of malaria resistance is largely ascribed by common SNPs and the causal variants are overrepresented in protein coding regions of the genome. Further studies with larger sample sizes are needed to better understand the underpinning genetics of severe malaria resistance.
\end{abstract}

\section{Introduction}

In spite of the global eradication efforts, malaria remains a major global public health problem with 219 million cases and 435000 deaths in 2017 (1). Plasmodium falciparum malaria results in diverse clinical manifestations ranging from asymptomatic parasitaemia to severe malaria (2). Such a wide variation of clinical outcome is attributed to several factors including genetic factors of the host, virulence of parasite and environmental factors (2). Family studies reported that the host genetic factors (heritability) contributes $\sim 25 \%$ of the variations observed in clinical severity of malaria in endemic populations (3). Thus, understanding the molecular basis of the natural immunity against severe malaria will speed up the development of an efficient malaria vaccine.

Driven by the wide availability of genome-wide single nucleotide polymorphism (SNP) arrays, the focus of genetic studies has been shifted from the traditional candidate gene and family-based linkage studies to GWASs. However, only a small fraction of narrow sense heritability is explained by the GWAS 
significant SNPs. This led to a problem commonly termed as 'missing heritability' (4). In effort to address this problem, several statistical methods that aim at quantifying SNP-heritability without identifying the causal variants were developed (5-7).

Packages such as the genome-wide complex trait analysis (GCTA) implement genetic relationship-matrix restricted maximum likelihood (GREML) method in which all SNPs of unrelated subjects are simultaneously analysed in a linear mixed model framework to estimate the proportion of phenotypic variations explained by genotypic variation (5). Apart from GREML approaches, Golan et al. (7) recently introduced a regression method called phenotype-correlation-genotype-correlation (PCGC) for estimation of heritability from case-control datasets. PCGC is a Haseman-Elston regression model in which a normalized phenotype is regressed on the genetic covariances of all unique pair of samples. The slope of the regression is used as an estimator of $h^{2}$ g. Application of these methods provided new insights in to the genetic architecture of complex diseases including autism, schizophrenia, Parkinson's disease, type 2 diabetes, hypertension among others (8-13). Alternative contemporary statistical methods that enable estimation of $h^{2}{ }_{g}$ from publicly available GWAS-summary statistics without the need of individual genotype data are also widely available (14-16) and gained popularity due to their privacy advantages and computational costs.

Because only unrelated individuals are included, the $h^{2}{ }_{g}$ analysis offers a greater flexibility of study designs that enable us to conduct powered studies. The use of only unrelated individuals also minimizes the biases from shared environments, one of the greatest challenges in pedigree studies (5). Furthermore, $h^{2}$ analytic methods allow partitioning of the cumulative heritability in to different functional categories and biological pathways and thus, provide more insights in to the underpinning biology $(6,17)$.

Even though a number of severe malaria GWASs have recently been implemented in endemic areas in Africa and reported few novel association variants (18-21), little is known about the $h^{2}$ and its distribution across the genome. Here we present results from a comprehensive $h^{2}{ }_{g}$ study of severe malaria resistance in three African populations including Gambia, Kenya and Malawi. We estimated $h^{2}{ }_{g}$ and partitioned in to chromosomes, different minor allele frequency (MAF) bins, functional categories and cell types. We found that the $h^{2}{ }_{g}$ is disproportionately concentrated on three chromosomes (chr 5, 11 and 20) and enriched in the coding region of genome. Overall, our results suggest that malaria resistance is mainly under polygenic control.

\section{Results}

\section{SNP-heritability estimates from genotype datasets}

We estimated $h^{2}{ }_{g}$ at different quality control (QC) levels to determine the appropriate threshold (see Materials and Methods). As expected, the estimates were inflated at less stringent QC thresholds (Supplementary Material, Fig. S1). However, applying stringent QC protocols including relatedness threshold (5\%), SNP differential missingness proportion $\left(P \leq 1 \times 10^{-3}\right)$ and SNPs missing proportion $(P>0.02)$ yielded a more stable ranges of $h^{2}{ }_{g}$ values that were not affected by the inclusion of additional principal components $(15,20,50)$ as covariates. At the stringent QC threshold, the $h^{2}{ }_{g}$ of severe malaria resistance was $0.21\left(\mathrm{se}=0.05, \mathrm{P}=2.7 \times 10^{-5}\right), 0.20\left(\mathrm{se}=0.05, \mathrm{P}=7.5 \times 10^{-5}\right)$ and 0.17 (se $\left.=0.05, P=7.2 \times 10^{-4}\right)$ in Gambian, Kenyan and
Malawi populations, respectively (Table 1). These estimates were approximately similar in Kenya ethnic groups such as Chonye $\left(0.20\right.$, se $\left.=0.07, P=5.1 \times 10^{-3}\right)$ and Giriama $(0.19$, se $=0.07$, $\left.P=7.3 \times 10^{-3}\right)$. However, the estimate was slightly inflated in Mandinka ethnic group of Gambia $\left(0.24\right.$, se $\left.=0.06, P=5.1 \times 10^{-5}\right)$. We did not estimate $h^{2}$ for other ethnic groups because of inadequate sample sizes. Furthermore, the PCGC model yielded broadly similar results including 0.20 , $\left(\mathrm{se}=0.06, P=9.7 \times 10^{-4}\right)$, $0.16\left(\mathrm{se}=0.06, \mathrm{P}=8 \times 10^{-3}\right)$ and $0.23\left(\mathrm{se}=0.07, \mathrm{P}=1.3 \times 10^{-3}\right)$ in Gambia, Kenya and Malawi populations, respectively.

Proportion of SNP-heritability attributable to GWAS loci. To quantify the effects of the known variants, we estimated the $h^{2}{ }_{g}$ without removing the severe malaria GWAS loci from the datasets (see Materials and Methods). This resulted in slight increment of the $h^{2}{ }_{g}$ estimate in Gambian [0.27 $\left(\mathrm{se}=0.05, \mathrm{P}=1 \times 10^{-5}\right)$ ] and Kenyan $\left[0.26\left(\mathrm{se}=0.05, \mathrm{P}<1 \times 10^{-5}\right)\right]$ populations. Repeating the GREML analysis by including $r \mathrm{~s} 334$ as an additional covariate decreased the estimate to $\left[0.24\left(\mathrm{se}=0.05, P<1 \times 10^{-5}\right)\right]$ and $\left[0.23\left(\mathrm{se}=5 \%, P<1 \times 10^{-5}\right)\right]$ in Gambian and Kenyan populations, respectively. This suggests that the $h^{2}{ }_{g}$ attributable to the GWAS significant loci and $\mathrm{HbS}$ locus is approximately 0.07 and 0.03 , respectively.

Partitioning SNP-heritability by chromosomes, minor allele frequencies and functional annotations. We observed no significant differences in $h^{2}$ g estimate obtained from separate analysis $\left(0.24\right.$, se $\left.=0.05, P<1 \times 10^{-5}\right)$ and the joint analysis $(0.22$, se $=0.05$, $P=1 \times 10^{-5}$ ) (Supplementary Material, Fig. S2), suggesting that the population structure was adequately controlled. Moreover, we observed significant correlations between chromosomal length and $h^{2}{ }_{g}$ per chromosome (Adj $r^{2}=0.38, P=0.001$ ) (Fig. 1). However, the estimates of three chromosomes (chr 5, 11 and 20) and three other chromosomes (chr 7, 8 and 15) fell above and below the expected $h^{2}{ }_{g}$ at $95 \%$ CI, respectively. Notably, chr5 contained a considerable proportion $(\sim 0.035)$ of the $h^{2}{ }_{g}$ (Supplementary Material, Table S1).

We performed MAF stratified analysis to estimate the relative contribution of variants with various allele frequencies (Fig. 2). However, we did not find significant differences between the proportion of $h^{2}$ attributed to different MAF bins [standards errors overlapped at $95 \%$ confidence interval (CI)]. Moreover, the total sum of our $h^{2}{ }_{g}$ estimate per bin [0.27 (se $\left.\left.=0.08, P=5.3 \times 10^{-5}\right)\right]$ was not significantly different from the univariate estimate. We further estimated the $h^{2}{ }_{g}$ explained by genic SNPs and the intergenic SNPs at $0.165(\mathrm{se}=0.05)$ and 0.062 (se $=0.05$ ), respectively (Table 2). On average, a SNP residing in genic region was enriched $2.9 \times$ compared to a SNP residing in an intergenic region of the genome. This is statistically significant at $95 \% \mathrm{CI}$.

\section{Functional enrichment from GWAS-summary statistics}

After imputation of severe malaria GWAS-summary statistics and QC filtering, we obtained a total of 20 million high quality SNPs (see Materials and Methods). Using this dataset, we estimated the liability scale $h^{2}{ }_{g}$ at 0.21 (se $\left.=0.02, P<1 \times 10^{-5}\right)$. Partitioning the $h^{2}$ gin to 24 main genomic annotations (baseline model) showed evidences of enrichment in multiple categories including $5^{\prime} \mathrm{UTR}(11 \times)$, digital genomic footprint (DGF; $10 \times)$, enhancer $(9 x)$, coding $(6 \times)$, H3K4me1 (4.9x), TSS ( $5 x)$, transcription factor binding sites (TFBS; $4 \times$ ) and FANTOM enhancer 
Table $1 . h^{2} g$ of severe malaria resistance determined by GCTA and PCGC methods

\begin{tabular}{lcccc}
\hline Population & SNPs $(n)$ & Samples $(n)$ & $h^{2}$ g-GCTA (\%) & $h^{2}$ g-PCGC (\%) \\
\hline Gambia & 1513822 & 4128 & $0.20(\mathrm{se}=0.05)$ & $0.20(\mathrm{se}=0.05)$ \\
Kenya & 1579227 & 2062 & $0.20(\mathrm{se}=0.05)$ & $0.16(\mathrm{se}=0.05)$ \\
Malawi & 1502462 & 2418 & $0.17(\mathrm{se}=0.05)$ & $0.23(\mathrm{se}=0.06)$ \\
Mandinka & 1513822 & 1281 & $0.24(\mathrm{se}=0.06)$ & ne* \\
Chonye & 1579227 & 637 & $0.20(\mathrm{se}=0.06)$ & ne* \\
Giriama & 1579227 & 1173 & $0.19(\mathrm{se}=0.06)$ & ne* \\
\hline
\end{tabular}

ne*: Model did not fit because of small sample size and there was no reliable estimation SNP: single nucleotide polymorphisms, $h^{2}{ }_{g \text {-GCTA }} h^{2}{ }_{g}$ estimated using GCTA method, $h^{2}{ }_{\text {PCGC }} h^{2}{ }_{g}$ estimated using PCGC method

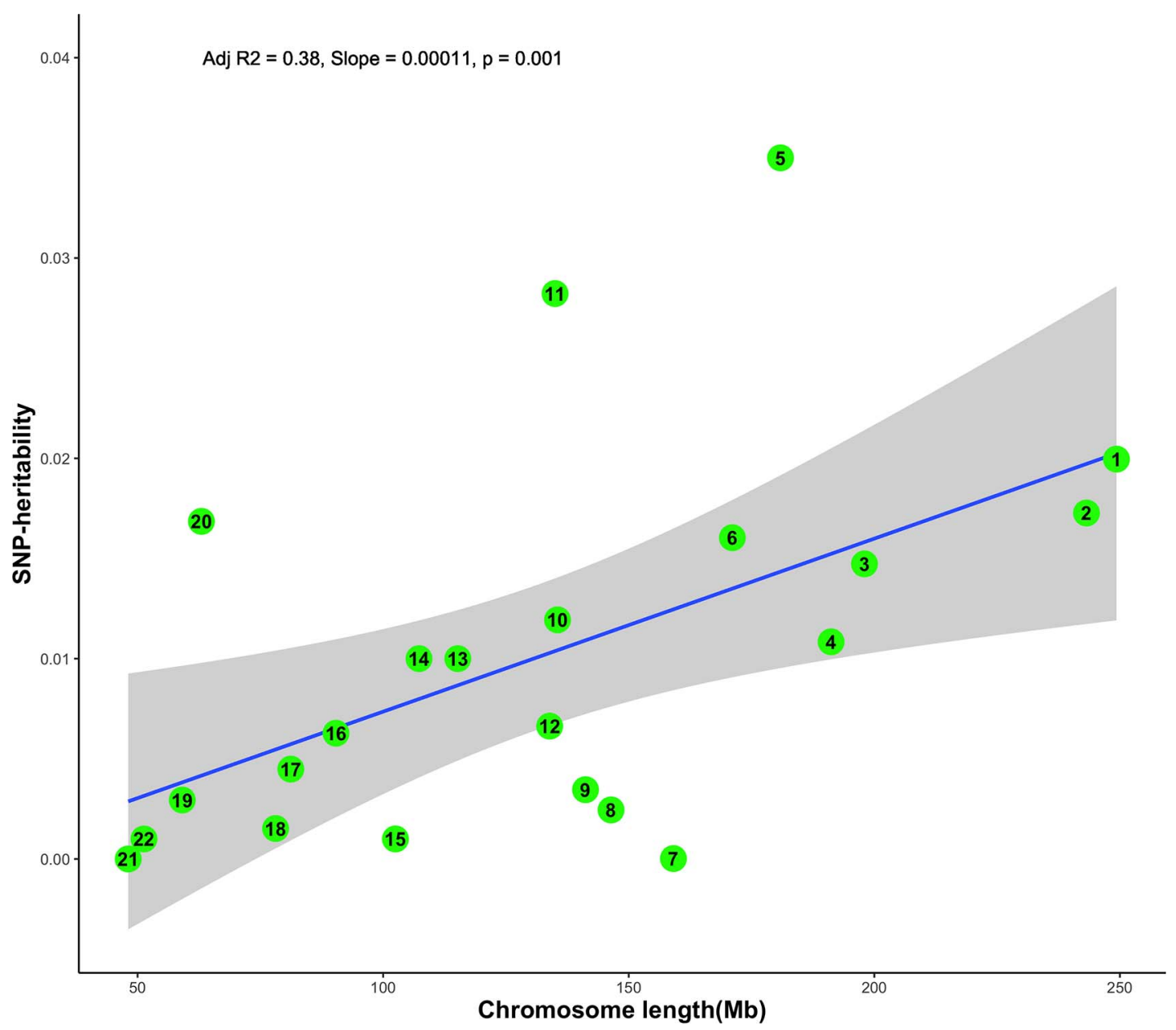

Figure 1. $h^{2} g$ per chromosome(y-axis) plotted against chromosome length (x-axis). The blue line represents the $h^{2} g$ estimates regressed against chromosome length. The grey shaded areas represent the $95 \% \mathrm{CI}$ around the slope of the regression model.

Table $2 . h^{2} g$ of severe malaria resistance partitioned in to genic and intergenic genomic regions

\begin{tabular}{lccc}
\hline SNP \\
location & $\operatorname{SNPs}(n)$ & $\begin{array}{c}10 \mathrm{~kb} \text { boundary } \\
h^{2} g\end{array}$ & $h^{2} g$ per SNP \\
\hline Genic & 727996 & $0.165(\mathrm{se}=0.05)$ & $2.3 \times 10^{-5}$ \\
Inter-genic & 785826 & $0.062(\mathrm{se}=0.05)$ & $7.9 \times 10^{-6}$ \\
\hline
\end{tabular}

$(4 \times)$ as shown in Figure 3. However, none of the enrichments was statistically significant after correction for multiple testing. Further cell-type specific and cell group analysis did not show significant enrichments.

\section{Discussions}

In this study, we estimated the $h^{2}{ }_{g}$ and functional enrichment of malaria resistance in three African populations and their meta-analysis. After excluding the severe malaria resistance GWAS loci, we performed GREML analysis at different QC levels to determine the appropriate threshold; indeed, the estimates were inflated upward at less stringent QC levels and became stable at more stringent QC levels. These estimates were broadly similar across the three study populations. Except a slight inflation observed in Mandinka ethnic group which might have been underpowered because of small sample size, the estimates were also similar across the major 


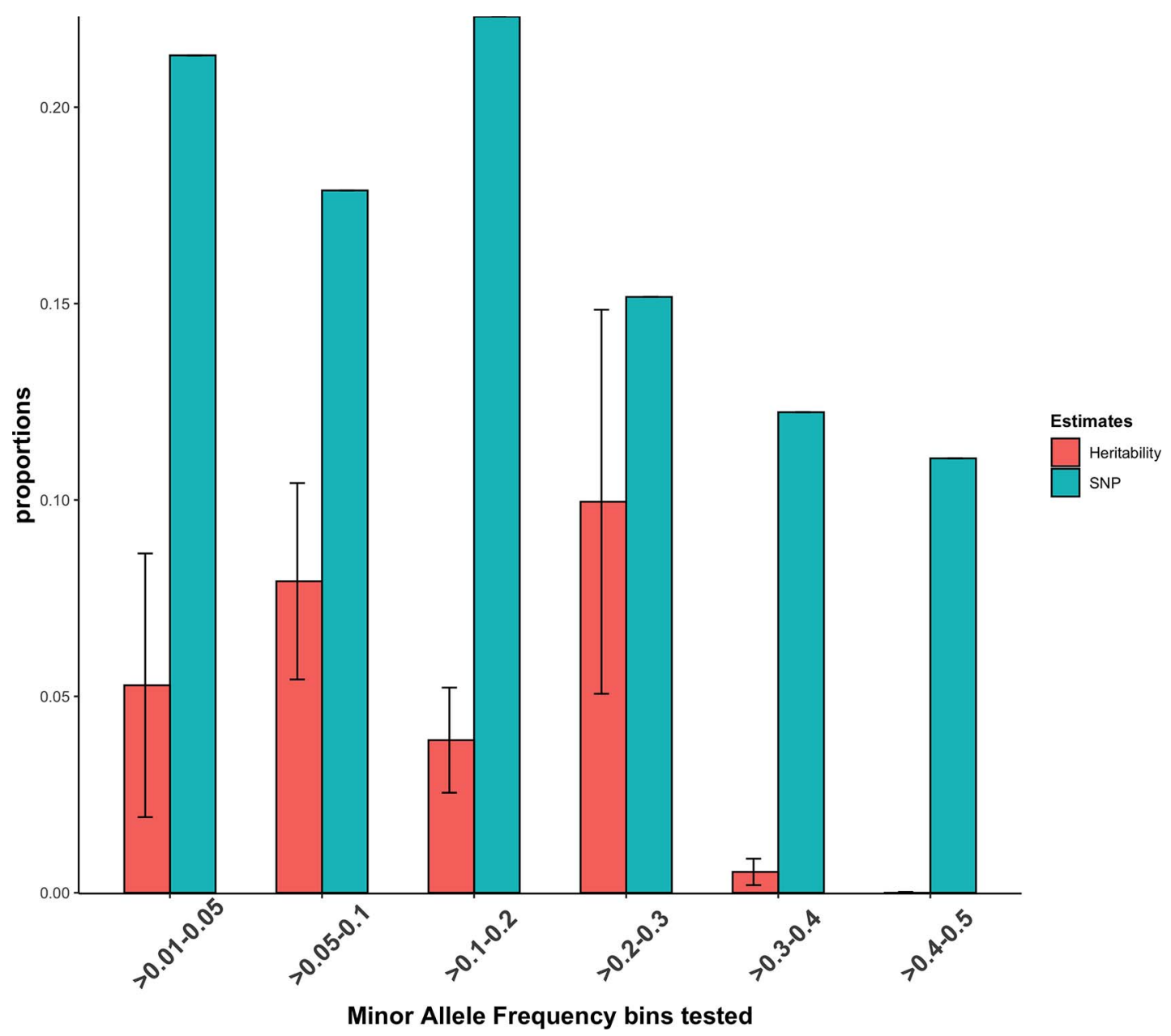

Figure 2. $h^{2} g$ partitioned in to different allele frequency spectrum. We created six MAF-bins and estimated the proportion of $h^{2} g$ attributed to each bin. The proportion of $h^{2} \mathrm{~g}$ attributed to each bin is shown in red bar and the proportion of SNPs per bin is shown by the blue bar. Error bars represent the $95 \% \mathrm{CI}$ of the estimate.

ethnic groups. Approximately a similar range of $h^{2}$ g of severe malaria resistance was recently reported (22). This might suggest that substantial human genetic factors that influence malaria disease severity have been maintained across endemic populations. Consistent with our findings, a previous familybased study reported a similar range of heritability of severe malaria resistance in two different endemic populations in Kenya (4).

In contrast to the findings from other complex disease studies in which $h^{2}$ is much smaller than family-based heritability values (23), our current $h^{2}$ g estimates were roughly close to a report from a previous family-based study (4). This might be due to the fact that the previous study underestimated the heritability estimates: First, only additive genetic effects (narrow-sense heritability) was calculated i.e. the contributions of nonadditive effects including epistasis, dominance and gene-gene interactions were not taken in to account. Second, the authors indicated that their paternity assessment was prone to misclassification, which might have underestimated the actual narrow-heritability estimate (4).

In the current study, including GWAS significant SNPs in the GREML analysis resulted in an increment of the $h^{2}{ }_{g}$ estimates by $\sim 0.07$ in the study populations, suggesting that the $h^{2}{ }_{g}$ attributable to the known malaria resistance GWAS loci ( $\left.h^{2}{ }_{g \text {-GWAS }}\right)$ is generally small. This is consistent with the hypothesis that the vast proportion of heritability of complex traits/diseases is explained by SNPs with effect sizes too small to attain the stringent genome wide significance threshold $\left(P<5 \times 10^{-8}\right)$ at the current sample sizes (24). Repeating the analysis by including rs334 as an additional covariate brought down these estimates by $\sim 0.03$, suggesting that more than a third of the $h^{2}{ }_{g \text {-GWAS }}$ is attributable to the HbS locus. This might be explained by the fact that the $\mathrm{HbS}$ locus has relatively larger effect sizes in the endemic populations (18).

To gain better insights in to the genetic architecture of severe malaria resistance, we partitioned the $h^{2}{ }_{g}$ in to different chromosomes, allele frequency spectrum and annotations. Separate GREML analysis and joint analysis yielded broadly similar $h^{2}{ }_{g}$ estimates, suggesting that population structure is adequately controlled. The rationale is that. However, the joint analysis in which genomic relatedness matrix (GRMs) of all chromosomes are simultaneously fitted in to a single GREML model, can effectively control the upward biases that can be created by correlated SNPs on different chromosomes (29).

Supporting the polygenic view of genetic architecture, we found a correlation between $h^{2}$ g per chromosome and chromosomal lengths (Adj $r^{2}=0.39, P=0.001$ ). However, the $h^{2}{ }_{g}$ is disproportionately concentrated on three chromosomes (chr 5 , 11 and 20) suggesting that these chromosomes might contain loci with larger effects against the polygenic background. Thus, 


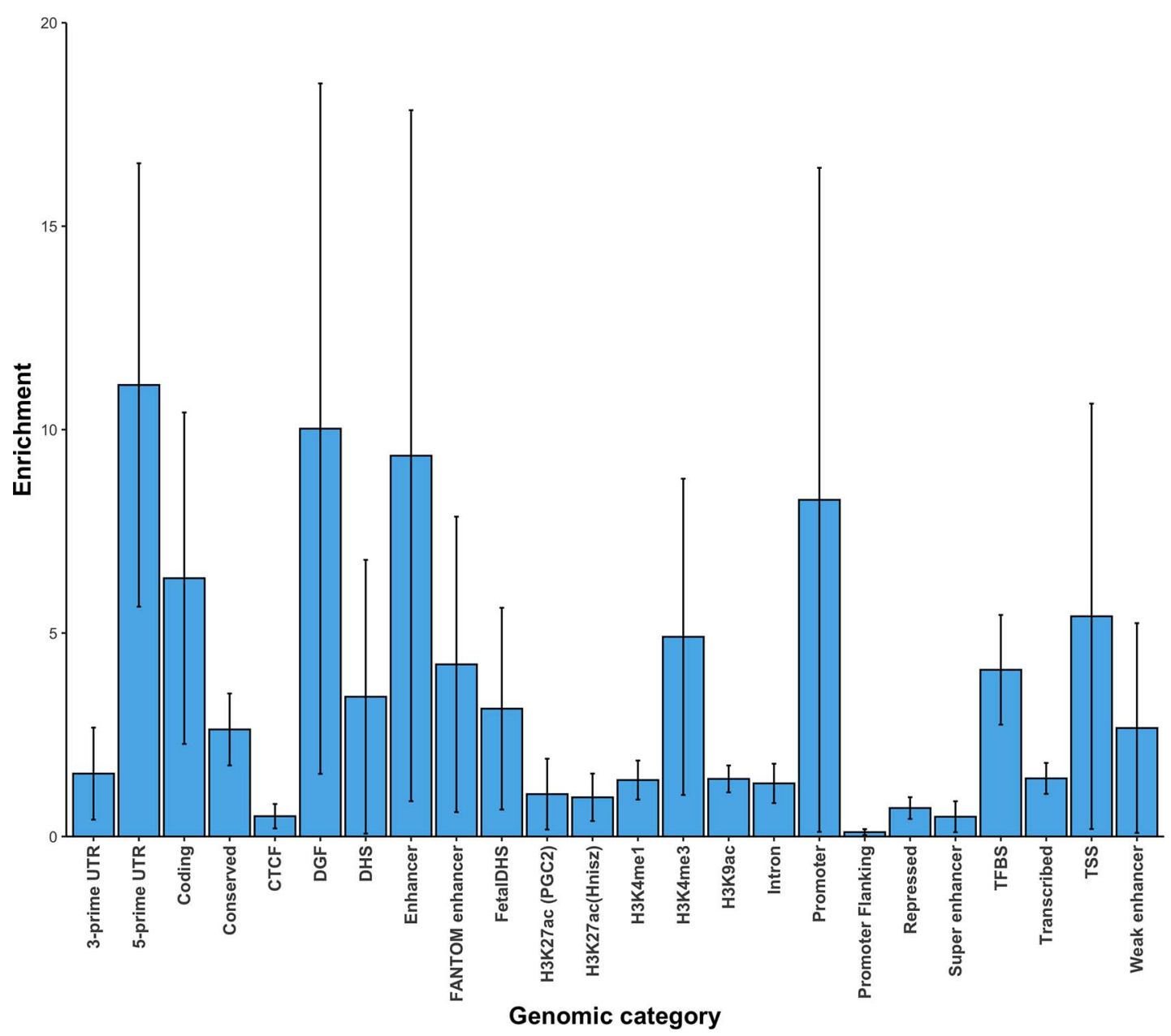

Figure 3. Enrichment estimates of $h^{2}$ g for the 24 main annotations. Error bars represent jackknife standard errors around the estimates of enrichment.

targeting these chromosomes using more powered studies (e.g. DNA sequencing) might be a cost-effective approach to discover new severe malaria resistance loci. Previous family-based studies reported that a region on chr5 (5q31-q33) is associated with susceptibility to mild malaria $(25,26)$.

MAF-stratified analysis didn't reveal significant differences between the proportion of $h^{2}$ attributed to different MAF bins. This might assert that $h^{2}$ g of severe malaria resistance is broadly uniform across the allele frequency spectrum and is not overrepresented by rare alleles. Partitioning by annotation revealed that the $h^{2}$ of severe malaria resistance is significantly enriched in SNPs residing in protein coding regions of the genome, suggesting that further studies focusing on coding regions (e.g. exome sequencing and/or exome array genotyping) might lead to the discovery novel variants.

In addition to the direct estimation of $h^{2}$ from raw genotype datasets, we performed functional enrichment analysis from GWAS-summary statistics using stratified linkage disequilibrium score regression (LDSC) approach (14). To improve the performance of the analysis, we created a reference panel that is more specific to our study populations by merging the African population datasets obtained from 1000 Genomes Project and African Genome Variation Project (27). Using this panel, we created annotation files and estimated $h^{2}$ of severe malaria resistance from GWAS-summary statistics meta-analysed across the study populations.
Our liability scale $h^{2}$, estimate $\left[0.21\left(\mathrm{se}=0.02, P<1 \times 10^{-5}\right)\right]$ was comparable to the direct estimates from raw genotype datasets. However, our functional enrichment analyses did not reveal significant results. One of the downsides of stratified LDSC method is that it requires very large sample sizes to detect significant enrichments (14). Of note, the coding genes and the surrounding categories were among the top annotations in our base line model. This further highlights the importance of protein coding regions of the genome in influencing the malaria disease severity.

Finally, our study had a number of caveats that might directly or indirectly affect the accuracy of estimating the true genetic heritability. First, the controls used in this study were not screened for mild malaria that might potentially bias the accuracy of $h^{2}{ }_{g}$ estimates. Second, assumptions of the models implemented for the analyses might not adequately explain the true genetic architecture of severe malaria resistance. Third, all the models implemented here do not measure the variances attributable to environmental factors. Fourth, the study is underpowered for the functional enrichment anlyses.

\section{Conclusions}

In conclusion, our study showed for the first time that heritability of severe malaria resistance is largely explained by common SNPs and is disproportionately enriched in SNPs residing 
in protein coding regions of the genome. Consistent with the polygenic genetic architecture, we observed that the $h^{2}{ }_{g}$ of severe malaria resistance is distributed across chromosomes and allele frequency spectrum. However, the $h^{2}{ }_{g}$ is disproportionately concentrated on three chromosomes (chr 5, 11 and 20), suggesting the cost-effectiveness of targeting these chromosomes in future malaria genomic sequencing studies. In this study, we created annotation files using population specific reference panel and showed that stratified LDSC analysis can provide reliable SNPheritability estimates in African populations. Further studies with larger sample sizes are needed to understand the unpinning genetics and biology of severe malaria resistance trait.

\section{Materials and Methods}

\section{Description of the study datasets}

GWAS datasets of three African populations including Gambia, Kenya and Malawi were obtained from European Phenome Genome Archive (EGA) through the MalariaGen consortium standard data access protocols $(28,29)$. The datasets contain information about a total of 11657 samples including 4921 samples from Gambia (2491 cases and 2430 controls), 3752 samples from Malawi (3752 cases and 3220 controls) and 2984 samples from Kenya (1506 cases and 1478 controls). Cases were obtained from children who were admitted to Hospitals and fulfilled WHO case definition for severe malaria (29) and controls were obtained from the general population (18-21). All the samples were genotyped on Illumina Omni $2.5 \mathrm{M}$ array. Information about phenotypes, imputation and QC was also provided.

\section{Quality control}

The basic QC protocols including plate effects, sample relatedness, Hardy-Weinberg equilibrium, heterozygosity, missingness and differential missingness were done as described elsewhere $(18,30)$. Taking in to consideration that small artifacts can have substantial cumulative effects in $h^{2}{ }_{g}$ analysis (31), we applied further stringent QC filtering steps. Briefly, we aligned the quality filtered VCF files to forward stand of the human reference sequence (GRCh3) using the illumina supplied files (www.well.ox.ac.uk/ wrayner/strand) and removed all SNPs with position and strand mismatches. We further removed SNPs with MAF below 0.01, deviate from Hardy-Weinberg at P-value below 0.01 using PLINK software (32). We then implemented step-wise QC filtering based on SNPs missingness proportion, differential missingness and sample relatedness as described in (6).

\section{Estimating heritability from genotype data}

We applied GCTA (5) and PCGC (7) models to estimate the $\mathrm{h}^{2}{ }_{\mathrm{g}}$ of severe malaria resistance from raw genotype datasets. Briefly, we excluded the region of extended inversion (7 $238552-$ 12442658 ) on chromosome 8p23 (33), the major histocompatibility complex (MHC) region (25000 000-40,000,000) on chr 6 and the known severe malaria resistance loci including the ATP2B 4 region on chr1:203154024-204154024, cluster of glycophorin (GYPA/B/E) region on chr4:143000000-146000000, ABO blood group region on chr9:135630000-136630 000, and the sickle cell (HbS) region on chr11:2 500 000-6500 000 to avoid potential biases from large effects.
We constructed GRMs from pruned high quality independent autosomal SNPs using GCTA software (5) and obtained list of samples with relatedness threshold $>5 \%$. We then computed GRMs using all the autosomal SNPs for each cohort and excluded one of any pair of samples with relatedness threshold $>5 \%$ as recommended elsewhere (6). The final sample of unrelated individuals was 4128, 2062 and 2418 for Gambia, Kenya and Malawi, respectively. The distribution of offdiagonal element of the GRMs for each population is shown in Supplementary Material, Fig. S3.

We used population prevalence of $1 \%$ of severe malaria as previously described in (29) and included the top 10 PCs as fixed effects in the GREML analysis. We then transformed the estimates to liability scale as described in Lee et al. (34). Using the same GRMs, we estimated the $h^{2}{ }_{g}$ using PCGC model as outlined in Golan et al. (12). We also computed separate GRMs and estimated $h^{2}$ for major ethnic groups in Gambia (Mandinka) and Kenya (Girimia and Chonye). Furthermore, we created GRMs in the presence of the GWAS significant SNPs and performed GREML analysis to quantify the effects of malaria resistance GWAS loci. We repeated the analysis by including rs334 as additional covariate to estimate the $h^{2}{ }_{g}$ attributable to $\mathrm{HbS}$.

Partitioning SNP-heritability from genotype data. Using Gambian dataset (largest sample size), we partitioned $\mathrm{h}^{2}$ by chromosomes, MAF bins and annotations. For the partitioning analyses, we excluded the severe malaria resistance GWAS loci to minimize the potential biases from SNPs with large effects. To investigate the biases that might be created by population structure, we performed separate and joint GREML analysis using all autosomal chromosomes. We first computed GRMs for individual autosomal chromosome and estimated $h^{2}{ }_{g}$ attributed to each chromosome by separate GREML in which one chromosome is fitted to the model at a time. We then performed a joint analysis in which GRMs of all autosomal chromosomes are simultaneously fitted in to a single GREML analysis and compared the results obtained from both analyses.

In addition to this, we partitioned the $h^{2}$ in to different allele frequencies and annotations. Briefly, we created five MAF bins including $>0.01-0.05,>0.05-0.1,>0.1-0.2,>0.2-0.3,>0.3-$ $04,>0.4-0.5$, computed separate GRMs for each bin and performed joint GREML analysis. For partitioning by annotation, we mapped all the autosomal SNPs to the human reference panel hg19 in UCSC genome database (http://genome.ucsc.edu) using QCTOOLV2 (https://www.well.ox.ac.uk/ gav/qctool) and obtained a list of genic and intergenic variants. Genic variants included those SNPs mapped to genomic regions within 10 kilobases $(\mathrm{kb})$ upstream and downstream of a protein coding gene. Intergenic variants included all the SNPs mapped to genomic regions outside $10 \mathrm{~kb}$ of a protein coding gene. We constructed separate GRMs and estimated $h^{2}{ }_{g}$ attributable to each category using the joint analysis implemented in GCTA software (5).

\section{Functional enrichment analysis of SNP-heritability from GWAS-summary statistics}

African-specific reference panel. Partitioning $h^{2}{ }_{g}$ in to cell-types and functional categories using stratified LDSC approach has recently been shedding new lights in to the genetic architecture of several complex diseases $(14,35,36)$. The method is based on the fact that a given category of SNPs is enriched for $h^{2}{ }_{g}$ if SNPs with high LD to that category have higher $\chi^{2}$ statistics than SNPs with low LD to that category $(35,36)$. However, the 
stratified LDSC analysis require population specific reference panel and very large sample sizes to produce reliable results (14). Consequently, the current European 1000G haplotype reference panel that is used as a default in LDSC software $(14,35)$ might not well represent our study populations.

To address this challenge, we created a reference panel that matches with our study populations. Briefly, we merged African population datasets obtained from 1000 Genomes Project and African Genome Variation Project (27) based on overlapped variants and removed structural variants and ambiguous SNPs using plink tool (32). This resulted in a combined dataset of sample size $(n=4975)$. After excluding the admixed populations including Americans of African Ancestry and African Caribbean, we clustered the dataset in to East African and west African sub-regions using smart pca software (37) as shown in Supplementary Material, Fig. S4. We removed SNPs with $\mathrm{MAF}<1 \%$, missingness $>0.05$ and HWE in controls (alpha level 0.0001), and retained a total of 22473268 SNPs (sample size $=2112$ ) and 18919068 SNPs (sample size $=380$ ) in east African and west African sub-regions, respectively. We finally calculated the MAF of the panel for later partitioning analysis. Owing to the fact that our study populations are comprised of both east African (Malawi and Kenya) and west Africa (Gambian) populations, we used the entire dataset as a reference panel for functional enrichment analysis.

Baseline model and functional annotations. We created baseline model and cell type specific annotations for our reference panel as described in (14). The baseline-LD model included 24 main annotations that are not cell type-specific including coding, UTRs ( $3^{\prime}$ UTR and 5'UTR), promoter and intronic regions obtained from UCSC genome browser and processed by Gusev et al. (12), the histone marks (H3) such as: acetylation of histone at lysine 9 (H3K9ac), monomethylation (H3K4me1) and trimethylation (H3K4me3) of $\mathrm{H} 3$ at lysine 4 obtained from Trynka et al. (38), acetylation of $\mathrm{H} 3$ at lysine $27(\mathrm{H} 3 \mathrm{~K} 27 \mathrm{ac}$ ) version one processed by Hnisz et al. (39) and version two Psychiatric Genomics Consortium, combined chromHMM and Segway predictions obtained from Hoffman et al. (40), regions that are conserved in mammals $(41,42)$, super enhancers (39), FANTOM5 enhancers (43), TFBS and DGF post-processed by Gusev et al (12). Around each partition, we added $500 \mathrm{bp}$ windows as separate categories to prevent biases that might arise from adjacent annotations.

The 24 main annotations together with the additional windows and a category containing all SNPs yielded 53 overlapping baseline model. Next, we created 220 cell type-specific annotations for the four histone marks: H3K4me1, H3K4me3, H3K9ac and H3K27ac (14) using our reference panel and computed LD score for each annotation. We then combined the 120 cell specific annotations in to 10 cell groups including adrenal and pancreas, central nervous system, cardiovascular, connective and bone, gastrointestinal, immune and hematopoietic, kidney, liver, skeletal muscle and other as described in (14). For each of the 10 categories, we computed the corresponding LD scores.

Stratified LDSC analysis. We obtained meta-analysed GWASsummary statistics of the three populations ( $n=15122094$ SNPs) from the previous GWAS (18). We performed imputation on this dataset using ImpG software (44). Briefly, we removed SNPs that mismatch with $1000 \mathrm{G}$ phase three markers, computed $z$ score from the association statistics and performed the imputation using ImGv.1.1 under default settings. We used all the 661 individuals labeled as 'AFRICAN' haplotypes in phase 1 of 1000
Genome Project version-3 calls (45). We removed all imputed SNPs with a predicted accuracy less than 0.9 and SNPs with $\mathrm{MAF}<0.01$. After QC filtering, we performed stratified LD score regression analysis using our reference panels as described in (14). Briefly, we converted the summary statistics to LDSC format, filtered SNPs with imputation accuracy greater than nine and MAF $>1 \%$, removed structural variants, ambiguous SNPs, the MHC region and significant SNPs. We then performed non-cell type- and cell type- specific analyses as described in (14).

\section{Funding}

National Research Foundation of South Africa for Funding (NRF) (grant RA171111285157/119056); The Centre for HighPerformance Computing (CHPC, www.chpc.ac.za) for computing resources; The Developing Excellence in Leadership and Genetics Training for Malaria Elimination in sub-Saharan Africa (DELGEME) program (grant PD00217ML to D.D.); NIH projects (to E.C.).

Conflict of Interest statement. The authors declare no competing interests.

\section{Authors Contributions}

DD designed, performed the data analysis and drafted the manuscript, EC contributed in designing, data-analysis and revision of the manuscript and supervised the work.

\section{Acknowledgements}

We thank Kwiatkowski's group from University of Oxford for their constructive comments and assistance. We thank Gavin Band for his advice and guidance in heritability analysis from genotype data. This work was supported through the DELTAS Africa Initiative [grant 107740/Z/15/Z]. The DELTAS Africa Initiative is an independent funding scheme of the African Academy of Sciences (AAS)'s Alliance for Accelerating Excellence in Science in Africa (AESA) and supported by the New Partnership for Africa's Development Planning and Coordinating Agency (NEPAD Agency) with funding from the Wellcome Trust [grant 107740/Z/15/Z] and the UK government. The views expressed in this publication are those of the author(s) and not necessarily those of AAS, NEPAD Agency, Wellcome Trust or the UK government.

\section{References}

1. World Health Organization (2018) World malaria report. World Health Organization, https://apps.who.int/iris/ handle/10665/275867.

2. Kwiatkowski, D.P. (2005) How malaria has affected the human genome and what human genetics can teach us about malaria. Am. J. Hum. Genet., 77, 171-192.

3. Mackinnon, M.J., Mwangi, T.W., Snow, R.W., Marsh, K. and Williams, T.N. (2005) Heritability of malaria in Africa. PLoS Med., 12, e340 10.1371/journal.pmed.0020340.

4. Manolio, T.A., Collins, F.S., Cox, N.J., Goldstein, D.B., Hindorff, L.A., Hunter, D.J., Mccarthy, M.I., Ramos, E.M., Cardon, L.R., Chakravarti, A. et al. (2009) Finding the missing heritability of complex diseases. Nature, 461, 747-753. 
5. Yang, J., Lee, S.H., Goddard, M.E. and Visscher, P.M. (2011) GCTA: a tool for genome-wide complex trait analysis. Am. J. Hum. Genet., 88, 76-82.

6. Speed, D., Cai, N., Johnson, M.R., Nejentsev, S. and Balding, D.J. (2017) Reevaluation of SNP heritability in complex human traits. Nat. Genet., 49, 986-992.

7. Golan, D., Lander, E.S. and Rosset, S. (2014) Measuring missing heritability: inferring the contribution of common variants. Proc. Natl. Acad. Sci., 111, E5272-E5281.

8. Klei, L., Sanders, S.J., Murtha, M.T., Hus, V., Lowe, J.K., Willsey, A.J., Moreno-de-luca, D., Yu, T.W., Fombonne, E., Geschwind, D. et al. (2012) Common genetic variants, acting additively, are a major source of risk for autism. Mol. Autism, 3(1), 1-13.

9. Keller, M.F., Saad, M., Bras, J., Bettella, F., Nicolaou, N., Sharma, M., Gibbs, J.R., Simo, J., Stefa, H., Heutink, P. et al. (2012) Using genome-wide complex trait analysis to quantify 'missing heritability' in Parkinson' s disease. Hum.Mol. Genet, 21, 4996-5009.

10. Loh, P.-R., Bhatia, G., Gusev, A., Finucane, H.K., BulikSullivan, B.K., Pollack, S.J., Schizophrenia Working Group of Psychiatric Genomics Consortium, de Candia, T.R., Lee, S.H., Wray, N.R. et al. (2015) Contrasting genetic architectures of schizophrenia and other complex diseases using fast variance-components analysis. Nat. Genet, 47, 1385-1392.

11. Lee, S.H., Yang, J., Goddard, M.E., Visscher, P.M. and Wray, N.R. (2012) Estimation of pleiotropy between complex diseases using single-nucleotide polymorphism-derived genomic relationships and restricted maximum likelihood. Bioinformatics, 28, 2540-2542.

12. Gusev, A., Lee, S.H., Trynka, G., Finucane, H., Vilhja, B.J., Xu, H., Zang, C., Ripke, S., Bulik-sullivan, B., Stahl, E. et al. (2014) Partitioning heritability of regulatory and cell-type-specific variants across 11 common diseases. Am. J. Hum. Genet., 95, 535-552 10.1016/j.ajhg.2014.10.004.

13. The Brian Consortium (2018) Analysis of shared heritability in common disorders of the brain. Science, 360, 8757-8769.

14. Finucane, H.K., Bulik-Sullivan, B., Gusev, A., Trynka, G., Reshef, Y., Loh, P.R., Anttila, V., Xu, H., Zang, C., Farh, K. et al. (2015) Partitioning heritability by functional annota- tion using genome-wide association summary statistics. Nat. Genet., 47, 1228-1235.

15. Weissbrod, O., Flint, J. and Rosset, S. (2018) Estimating heritability and GeneticCorrelation in case control studies directly and with summary statistics. Am. J. Hum. Genet., 103, 89-99 10.1016/j.ajhg.2018.06.002.

16. Speed, D. and Balding, D.J. (2019) SumHer better estimates the SNP heritability of complex traits from summary statistics complex traits from summary statistics. Nat. Genet., 51, 277-284.

17. Visscher, P.M., Macgregor, S., Benyamin, B., Zhu, G., Gordon, S., Medland, S., Hill, W.G., Hottenga, J., Willemsen, G., Boomsma, D.I. et al. (2007) Genome partitioning of genetic variation for height from 11, 214 sibling pairs. Am. J. Hum. Genet., 81, 1104-1110.

18. Band, G., Rockett, K.A., Spencer, C.C.A., Kwiatkowski, D.P., Band, G., Si Le, Q., Clarke, G.M., Kivinen, K., Leffler, E.M., Rockett, K.A. et al. (2015) A novel locus of resistance to severe malaria in a region of ancient balancing selection. Nature, 526, 253-257.

19. Timmann, C., Thye, T., Vens, M., Evans, J., May, J., Ehmen, C., Sievertsen, J., Muntau, B., Ruge, G., Loag, W. et al. (2012) Genome-wide association study indicates two novel resistance loci for severe malaria. Nature, 489, 443-446.
20. Jallow, M., Teo, Y.Y., Small, K.S., Rockett, K.A., Deloukas, P., Clark, T.G., Kivinen, K., Bojang, K.A., Conway, D.J., Pinder, M. et al. (2009) Genome-wide and fine-resolution association analysis of malaria in West Africa. Nat Genet, 41, 657-665 $10.1038 / \mathrm{ng}$.

21. Ravenhall, M., Campino, S., Sepu, N., Nadjm, B., Mtove, G., Wangai, H., Maxwell, C., Olomi, R., Reyburn, H., Drakeley, C.J. et al. (2018) Novel genetic polymorphisms associated with severe malaria and under selective pressure in North-Eastern Tanzania. PLoS Genet., 14, e1007172 https://doi.org/10.1371/journal. pgen.1007172.

22. Malaria Genomic Epidemiology Network Consortium (2019) New insights into malaria susceptibility from the genomes of 17,000 individuals from Africa, Asia, and Oceania. bioRxiv. http://dx.doi.org/10.1101/535898.

23. Mayhew, A.J. and Meyre, D. (2017) Assessing the heritability of complex traits in humans: methodological challenges and opportunities. Current Genom, 18, 332-340 10.2174/1389202918666170307161450.

24. Visscher, P.M., Brown, M.A., Mccarthy, M.I. and Yang, J. (2012) Five years of GWAS discovery. Am. J. Hum. Genet., 90, 7-24.

25. Flori, L., Sawadogo, S., Esnault, C., Fre, N., Fumoux, F. and Rihet, P. (2003) Linkage of mild malaria to the major histocompatibility complex in families living in Burkina Faso. Hum. Mol. Genet., 12, 375-378.

26. Brisebarre, A., Kumulungui, B., Sawadogo, S., Atkinson, A., Garnier, S., Fumoux, F. and Rihet, P. (2014) A genome scan for plasmodium falciparum malaria identifies quantitative trait loci on chromosomes 5q31, 6p21.3, 17p12, and 19p13. Malar. J., 13, 1-7.

27. Gurdasani, D., Carstensen, T., Tekola-Ayele, F., Pagani, L., Tachmazidou, I., Hatzikotoulas, K., Karthikeyan, S., Iles, L., Pollard, M.O., Choudhury, A. et al. (2015) The African genome variation project shapes medical genetics in Africa. Nature, 517, 327-332.

28. Parker, M., Bull, S.J., Vries, J., Agbenyega, T., Doumbo, O.K. and Dominic, P. (2009) Ethical data release in genome-wide association studies in developing countries. PLoSMed, 6, 11e1000143 10.1371/journal.pmed.1000143.

29. Achidi, E.A., Agbenyega, T., Allen, S., Amodu, O., Bojang, K., Conway, D., Corran, P., Deloukas, P., Djimde, A., Dolo, A. et al. (2008) A global network for investigating the genomic epidemiology of malaria. Nature, 456, 732-737.

30. Band, G., Le, Q.S., Jostins, L., Pirinen, M., Kivinen, K., Jallow, M., Sisay-Joof, F., Bojang, K., Pinder, M., Sirugo, G. et al. (2013) Imputation-based meta-analysis of severe malaria in three African populations. PLoS Genet, 10.1371/journal.pgen.1003509.

31. Speed, D., Hemani, G., Johnson, M.R. and Balding, D.J. (2012) Improved heritability estimation from genome-wide SNPs. Am. J. Hum. Genet., 91, 1011-1021.

32. Purcell, S., Neale, B., Todd-Brown, K., Thomas, L., Ferreira, M.A.R., Bender, D., Maller, J., Sklar, P., de, P.I.W., Daly, M.J. et al. (2007) PLINK: a tool set for whole-genome association and population-based linkage analyses. Am. J. Hum. Genet., 81, 559-575.

33. Antonacci, F., Kidd, J.M., Marques-bonet, T., Ventura, M., Siswara, P., Jiang, Z. and Eichler, E.E. (2009) Characterization of six human disease-associated inversion polymorphisms. Hum. Mol. Genet., 18, 2555-2566 10.1093/hmg/ddp187.

34. Lee, S.H., Wray, N.R., Goddard, M.E. and Visscher, P.M. (2011) Estimating missing heritability for disease from genome-wide association studies. Am. J. Hum. Genet., 88, 294-305. 
35. Jiang, X., Finucane, H.K., Schumacher, F.R., Schmit, S.L., Tyrer, J.P., Han, Y., Michailidou, K., Lesseur, C., Kuchenbaecker, K.B., Dennis, J. et al. (2019) Shared heritability and functional enrichment across six solid cancers. Nat.Commun, 10, 431-454 10.1038/s41467-018-08054-4.

36. Gazal, S., Finucane, H.K., Furlotte, N.A., Loh, P. and Palamara, P.F. (2017) Linkage disequilibrium dependent architecture of human complex traits shows action of negative selection. Nat Genet., 49, 1421-1427.

37. Trynka, G., Sandor, C., Han, B., Xu, H., Stranger, B.E., Liu, X.S. and Raychaudhuri, S. (2013) Chromatin marks identify critical cell types for fine mapping complex trait variants. Nat Genet., 45, 124-130 10.1038/ng.2504.

38. Sigova, A.A., Hnisz, D., Abraham, B.J., Lee, T.I., Lau, A., Saintandre, V., Hoke, H.A. and Young, R.A. (2013) Resource superenhancers in the control of cell identity and disease. Cell., 155, 934-947.

39. Hoffman, M.M., Ernst, J., Wilder, S.P., Kundaje, A., Harris, R.S., Libbrecht, M., Giardine, B., Ellenbogen, P.M., Bilmes, J.A., Birney, E. et al. (2013) Integrative annotation of chromatin elements from ENCODE data. Nuc.Ac.Res., 41, 827-841.
40. Lindblad-toh, K., Garber, M., Zuk, O., Lin, M.F., Parker, B.J., Washietl, S., Kheradpour, P., Ernst, J., Jordan, G., Mauceli, E. et al. (2011) A high-resolution map of human evolutionary constraint using 29 mammals. Nature, 478, 476-481.

41. Ward, L.D. and Kellis, M. (2012) Evidence of abundant purifying selection in humans for recently acquired regulatory functions. Sci., 337, 1675-1683.

42. Andersson, R., Gebhard, C., Miguel-escalada, I., Hoof, I., Bornholdt, J., Boyd, M., Chen, Y., Zhao, X., Schmidl, C., Suzuki, T. et al. (2014) An atlas of active enhancers across human cell types and tissues. Nature, 507, 455-461 10.1038/nature12787.

43. Pasaniuc, B., Zaitlen, N., Shi, H., Bhatia, G., Gusev, A., Pickrell, J., Hirschhorn, J., Strachan, D.P., Patterson, N. and Price, A.L. (2014) Fast and accurate imputation of summary statistics enhances evidence of functional enrichment. Bioinformatics, 30, 2906-2914 10.1093/bioinformatics/btu416.

44. The 1000 Genomes Project Consortium (2011) A map of human genome variation from population scale sequencing. Nature, 467, 1061-1073. 